\title{
Theoretical study of polar cap arcs: Time-dependent model and its applications
}

\author{
L. Zhu, J. J. Sojka, R. W. Schunk, and D. J. Crain \\ Center for Atmospheric and Space Sciences, Utah State University, Logan
}

A time-dependent theoretical model of polar cap arcs developed during the Coupling, Energetics, and Dynamics of Atmospheric Regions/High-Latitude Plasma Structures (CEDAR/HLPS) campaigns in the past two years is briefly described. In the model the electrodynamics of the polar cap arcs are treated self-consistently in the frame of the coupled magnetosphere-ionosphere system. The preliminary simulation results of the temporal evolution and spatial structure of the polar cap arcs for both winter and summer conditions are presented. The model can be used to conduct both the model-observation study of specific features of the polar cap arcs and the quantitative theoretical study of typical or average features of the arcs. Several potential specific applications of the model to the polar cap arc phenomenon are also discussed in the paper.

\section{INTRODUCTION}

A series of campaigns conducted under the auspices of the Coupling, Energetics, and Dynamics of Atmospheric Regions/High-Latitude Plasma Structure (CEDAR/HLPS) Working Group in the past two years have accumulated a wealth of information on both the interplanetary magnetic field (IMF) southward patch phenomena and the IMF northward polar cap arc phenomena, and the quantitative theoretical modeling and self-consistent model-observation study of these phenomena have now become feasible. Here we show preliminary simulation results of polar cap arcs for both winter and summer conditions from a time-dependent theoretical model developed by Zhu et al. [1993] at Utah State University during the HLPS campaigns in the past two years and describe the capability of the model for coordinated model-observation study and theoretical modeling and the potential applications of the model to several specific phenomena associated with polar
cap arcs.

The polar cap arcs were mainly observed during the periods of northward IMF and quiet magnetic conditions. Some of the arcs were very bright and luminous, which crossed the polar cap from the dayside to the nightside of the auroral oval to form a pattern of Greek letter "theta" [Frank et al., 1986; Nielsen et al., 1990], while the others were confined in the polar cap and relatively weak, which had multiple discrete patterns and were either in the evening sector or morning

\footnotetext{
Copyright 1994 by the American Geopysical Union

Paper number $93 \mathrm{RS} 01508$.
$0048-6604 / 94 / 93 R S-01508$

0048-6604/94/93RS-01508\$08.00
}

sector of the polar cap [Weber and Buchau, 1981; Carlson et al., 1984; Rich et al., 1990]. Some observations suggest that polar cap arcs are in the regions of open field lines because polar rain electrons were observed adjacent to and outside of the arcs [Hardy et al., 1982]. In other observations the precipitating electrons having the characteristics of plasma sheet or plasma sheet boundary layer electrons were observed, suggesting that the arcs are on the closed field lines [Obara et al., 1988]. The convection patterns associated with the arcs could be mesoscale shear flow patterns [Carlson et al., 1988; Valladares and Carlson, 1991], or they could be ambiguous and confused ones [Rich et al., 1990]. One general consensus in the observations of polar cap arcs is that the arcs are associated with upward field-aligned currents which are carried by energetic precipitating electrons, and the energy range of the precipitating electrons spans from a few hundred of $\mathrm{eV}$ to a few keV [Hardy et al., 1982; Valladares and Carlson, 1991]. The dependence of features of polar cap arcs on the $Y$ component of the IMF has also been reported by many researchers [e.g., Makita et al., 1991]. Most recently, several interesting features of polar cap arcs were reported at the Workshop for Coupling Energetics and Dynamics of Atmospheric RegionsHigh Latitude Plasma Structures held at Peaceful Valley, Colorado. Valladares [1992] reported the dawn-dusk motion of polar cap arcs. The speed of such a motion can be as high as $500 \mathrm{~m} \mathrm{~s}^{-1}$, and he suggested that the dawn-dusk motion of polar cap arc might be $B$-related. Berg [1992] found that prior to the breakup of polar cap arcs, the adjacent convection underwent a sudden rotation. The field-aligned depletions of $F$ region plasma associated with polar cap arcs have been reported by Doe et al. [this issue]. 
Compared to the observations, the theoretical studies of polar cap arcs, especially the quantitative theoretical studies, are still in their early stages. One recent development in this aspect is the two parallel theoretical model studies of polar cap arcs being conducted by the Utah State University research group. One is an effort to study the ionospheric response to the magnetospheric convection and precipitation pattern associated with polar cap arcs. By using the time-dependent ionospheric model (TDIM) [Schunk and Sojka, 1982], the study can provide a three-dimensional quantitative picture of the ionospheric features associated with polar cap arcs. The details of the study of polar cap arcs using TDIM is described by Crain et al. [this issue]. The other is an effort to study the electrodynamics and underlying physics of polar cap arcs in the frame of the coupled magnetosphere-ionosphere system. A time-dependent model of polar cap arcs has been developed along this direction [Zhu et al., 1993], and the major components of the model will be summarized in this paper. The key point in this approach is that polar cap arcs are not treated as the phenomena merely controlled by the magnetosphere acting as a driver, with the ionosphere acting as a passive load; instead, the polar cap arcs are treated as the physical processes which are coherently determined by the dynamics in both the ionosphere and magnetosphere.

The paper will proceed in the following way. The physics, mathematical formulation, and numerical aspect of the model are briefly reviewed in section 2 (for the details of the model formulation, see Zhu et al. [1993]). In section 3, some preliminary modeling results are shown and discussed. Then we describe the capability and potential applications of the developed model in section 4 .

\section{A BRIEF REVIEW OF THE MODEL}

Polar cap arcs are neither isolated ionospheric physical processes nor passive ionospheric manifestations of the dynamics occurring in the magnetosphere. The dynamical features of polar cap arcs are coherently determined by the dynamics in the magnetosphere and the ionospheric conditions, in which the ionosphere is not just a passive medium or a "load." This suggests that a self-consistent study of polar cap arcs can be undertaken only in the frame of a coupled magnetosphere-ionosphere (M-I) system. But on the other hand, the coupled M-I system is very complicated and involves a number of dynamical processes. At this stage a comprehensive quantitative model, which includes all dynamical processes in the M-I system and can also show the detailed features of polar cap arcs, is almost impossible. As a first step, Zhu et al. [1993] have formulated a quantitative model of polar cap arcs, which is kept as simple as possible, but contains key physical processes which are treated selfconsistently in the frame of the coupled M-I system. The scenario of processes in the model is described as follows.

Figure 1 is a schematic diagram showing the M-I framework for our polar cap arc model. Initially, a magnetospheric shear flow carried by Alfvén waves propagates toward the ionosphere. The significance of the assumed initial
magnetospheric shear flow in the model has been discussed magnetospheric shear flow in the model has been discussed by Zhu et al. [1993]. The downward propagating Alfvén waves are partially reflected from the ionosphere and then bounce around between the ionosphere and magnetosphere. The features of wave reflections depend on the conditions in the ionosphere and magnetosphere. The propagating Alfvén waves carry both upward and downward field-aligned currents. The precipitating electrons associated with upward field-aligned currents enhance the conductivity in the ionosphere. The changed ionospheric conductivity launches a secondary Alfvén wave toward the magnetosphere. The whole process is transient during which all physical quantities in the ionosphere change self-consistently in time and polar cap arcs develop. Because of the finite conductivity in the ionosphere, the temporal change of Alfvén waves in the coupled M-I system diminish with time, and the whole M-I system, as well as the development of polar cap arcs approaches an asymptotic steady state.

The ionosphere is treated as a two-dimensional slab with an integrated conductivity. The $X$ axis measures the noonmidnight dimension and points to the dayside, whereas the $Y$ axis measures the dawn-dusk dimension and points to the duskside. The magnetosphere is a two-dimensional pseudoplane used as a boundary for the reflection of Alfvén waves. The magnetosphere and ionosphere are connected by magnetic field lines which are assumed perpendicular to the ionosphere. The communication between the magnetosphere and ionosphere is characterized by the bouncing of Alfvén waves between the two regions, and both the upward and downward field-aligned currents are associated with the propagating Alfvén waves. Initially, the large-scale ionospheric convection and conductivity are given. These large-scale patterns are consistent with the overall solar, geomagnetic, and IMF conditions. The background convection shown in Figure 1 is not necessarily a two-cell convection, it has only been selected for the purpose of illustration. The ionospheric simulation domain can be either on open field lines, closed field lines, or on both, depending on the choice of the wave reflection character at the magnetospheric boundary. The initial time $(t=0 \mathrm{~min})$ is the time when the magnetospheric shear flow carried by Alfvén waves first arrives at the ionosphere.

The propagating Alfvén waves can carry both field-aligned currents and transverse polarization currents. The relationship currents and transverse polarization currents. The relationsived
between the wave electric field and the currents can be derive from MHD equations and expressed as

$$
\mathbf{J}_{A}= \pm \Sigma_{A}\left[-\mathbf{b}_{o}(\nabla \cdot \mathbf{E})+\left(\mathbf{b}_{o} \cdot \nabla\right) \mathbf{E}\right]
$$

where $\mathbf{b}_{O}$ is the unit vector of the magnetic field, $\Sigma_{A}=$ $\left(\mu_{O} V_{A}\right)^{-1}$ is the characteristic conductance of the plasma field-aligned current carried by Alfvén wave, and the $s$ 


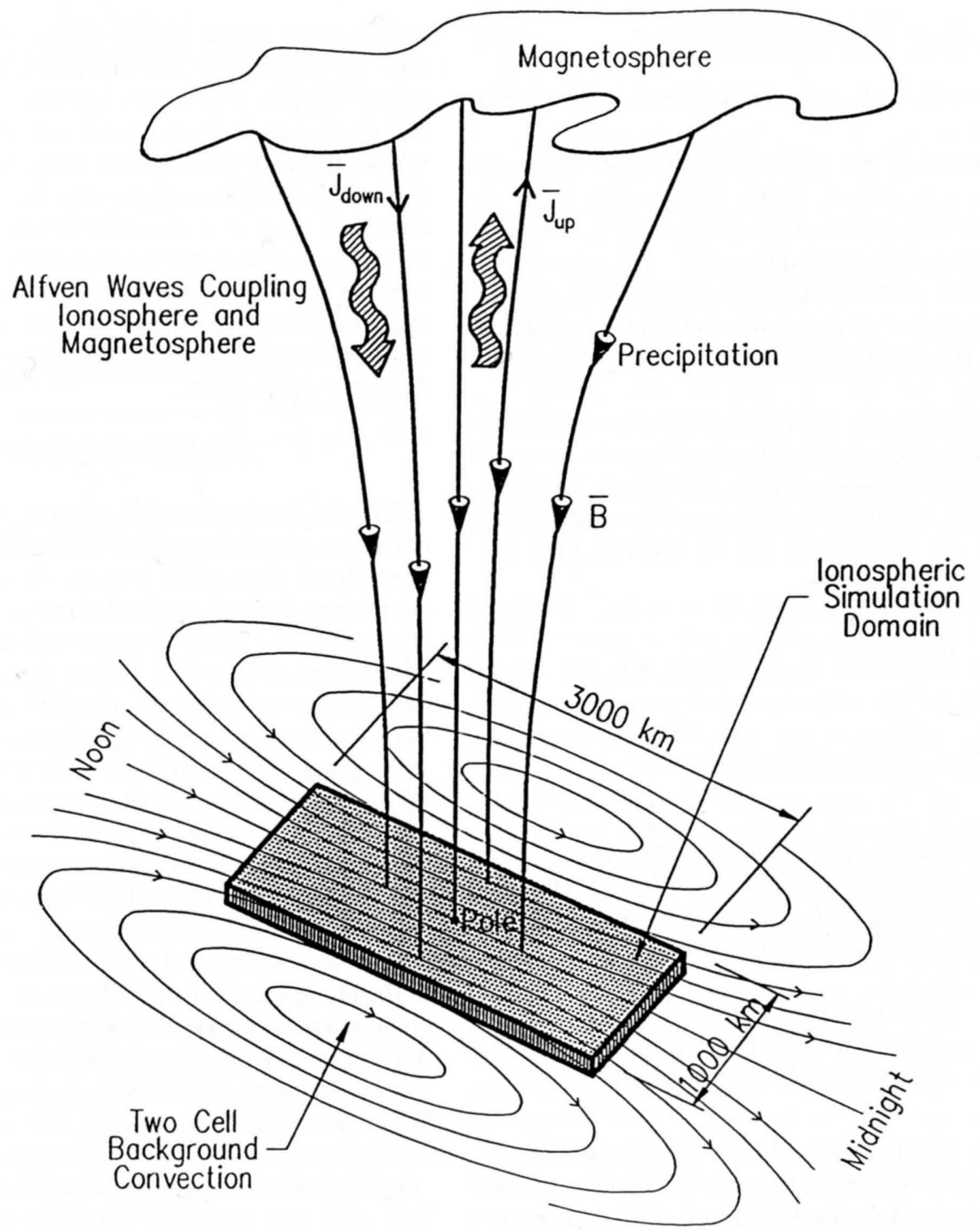

Fig. 1. A schematic diagram showing the geophysical framework of the polar cap arc model. The background ionospheric convection is used for illustrative purposes only and is not necessarily a two-cell convection.

term is the polarization current flowing across field lines along
the wavefront.
By matching the field-aligned currents carried by the incident
and the reflected waves and the field-aligned currents which
exist in front of the wave front of the Alfvén waves $\left(J_{\| D}\right)$ with
the divergence of the ionospheric currents, we obtain a time-
dependent equation which describes the reflection of an Alfvén
wave from the ionosphere:

$$
\begin{aligned}
& \Sigma_{A} \nabla \cdot\left[\mathbf{E}^{i}(t)-\mathbf{E}^{r}(t)\right]+J_{\| D}(t) \\
& =\nabla \cdot\left[\Sigma_{P}(t) \mathbf{E}_{i}(t)+\Sigma_{H}(t) \mathbf{b}_{o} \times \mathbf{E}_{i}(t)\right]
\end{aligned}
$$

and

$$
\mathbf{E}_{i}(t)=\mathbf{E}^{i}(t)+\mathbf{E}^{r}(t)+\mathbf{E}_{i P}(t)
$$

where $\mathbf{E}^{\boldsymbol{i}}$ is the incident wave field. $\mathbf{E}^{\boldsymbol{r}}$ is the reflected wave field, $\mathbf{E}_{\boldsymbol{i}}$ is the total ionospheric electric field, $\mathbf{E}_{\boldsymbol{i}}$ is the ionospheric electric field which excludes the incident and the reflected wave fields, and $\Sigma_{P}$ and $\Sigma_{H}$ are the ionospheric Pedersen conductance and Hall conductance, respectively.

On the magnetospheric side, a reflection coefficient is used to describe the reflection of the upward traveling Alfvén waves at the magnetospheric boundaries, which can be expressed as 


$$
\begin{gathered}
R_{m}=-1 \text { on open field lines } \\
-1<R_{m}<1 \text { on closed field lines }
\end{gathered}
$$

The physics governing the reflection coefficient has been discussed by Zhu et al. [1993]. It may be noted that $R_{m}=-1$ corresponds to an idealized constant voltage source, while $R_{m}$ $=1$ corresponds to a constant current source. The value of $R_{m}$ on closed field lines can be expected to increase from slightly greater than -1 toward +1 as one moves from the distant tail or the low-latitude boundary layer toward the inner edge of the plasma sheet.

Under the assumption that precipitating electrons associated with upward field-aligned currents are the main external ionization source in the polar cap in addition to solar UV contributions, the equation describing the conductivity enhancement in the ionosphere can be derived from the continuity equation, which is

$$
\begin{aligned}
& \frac{\partial \Sigma_{H}}{\partial t}=\frac{(Q H+1)}{B_{o}} \nabla \cdot\left(\Sigma_{P} \mathbf{E}_{i}+\Sigma_{H} \mathbf{b}_{o} \times \mathbf{E}_{i}\right) \\
& +\frac{\mathrm{e} H}{\boldsymbol{B}_{o}} \mathrm{~S}_{\mathrm{o}}-\frac{\beta B_{o}}{\mathrm{e} H} \Sigma_{H}^{2}+\nabla \Sigma_{H} \cdot \frac{\mathbf{b}_{o} \times \mathbf{E}_{i}}{B_{o}}
\end{aligned}
$$

where $H$ is the effective height of the conducting ionosphere, $B_{O}$ is the background magnetic field, $S_{O}$ is the background ionization source term, $\beta$ is the recombination coefficient, and $Q$ is the ionization coefficient which is defined as

$$
\begin{array}{ll}
Q=0 & J_{\|} \text {downward or }\left|J_{\|}\right|<J_{O} \\
Q=Q_{O} & J_{\|} \text {upward and } \nabla \cdot \mathbf{E}_{i} \geq 0 \text { and }\left|J_{\|}\right|>J_{O} \\
Q=\gamma^{\prime}\left(\left|J_{\|}\right|-J_{O}\right) & J_{\|} \text {upward and } \nabla \cdot \mathbf{E}_{i}<0 \text { and }\left|J_{\|}\right|>J_{O}
\end{array}
$$

where $Q_{o}$ is a constant depending on the energy of precipitating electrons which are not associated with potential drop structures along magnetic field lines, $\gamma^{\prime}$ is a parameter which depends on the $J_{\|}-\phi \|$ relationship [Fridman and Lemaire, 1980], and $J_{O}$ is the thermal electron current in the loss cone. The ionization coefficient $Q$ is defined as the average number of ions produced by an incident electron per meter, which is proportional to the energy of the precipitating electrons.

Equations (2), (3), (4), and (5) form the basic working equations of the present model of polar cap arcs. The temporal evolution of polar cap arcs and Alfvén waves propagating along magnetic field lines can be obtained by solving these equations numerically.

The ionospheric simulation domain is $3000 \mathrm{~km}$ long in the $X$ (midnight-noon) direction, and $1000 \mathrm{~km}$ wide in the $Y$ (dawn-dusk) direction. The grid size is $30 \mathrm{~km}$ in the $X$ direction, and $10 \mathrm{~km}$ in the $Y$ direction. The third dimension along the magnetic field lines is a pseudodimension which merely serves to provide the Alfvén wave traveling time scale. The magnetosphere is simplified as a plane which is the source region of the initial magnetospheric shear flow and is used only for treating the reflection of the Alfvén waves on the magnetospheric side after $t=0 \mathrm{~min}$. The polar cap arcs are mainly aligned in the noon-midnight direction. In the distant regions on the morningside or duskside of the polar cap arcs the background ionosphere can be assumed undisturbed; therefore we use a constant boundary condition for the dawnside and duskside boundaries in the simulation. This constant boundary condition may stabilize the simulation. An open boundary condition is used for the noonside and midnightside boundaries, which allows plasma to freely flow through the boundaries.

\section{PRELIMINARY RESULTS}

With the developed model, we can conduct theoretical studies of the typical or average features of polar cap arcs by using the generalized theoretical inputs, or we can make modelobservation study of specific features and specific events of the arcs by directly using observational data as the inputs. The initial inputs to the model include the ionospheric background conductivity reflecting the solar and seasonal conditions, the large-scale ionospheric background convection, and the smallscale shear flow associated with polar cap arcs. In the following paragraphs, we describe some preliminary results of the temporal evolution and spatial structure of polar cap arcs from the simulations using the generalized theoretical inputs. The results of two theoretical runs are shown in this paper. One is for summer, solar maximum conditions, and the other is for winter, solar maximum conditions.

The top panel of Figure 2 shows the initial Hall conductance distributions in the noon-midnight cross section for these two runs. Curve A is for summer conditions, and curve B is for winter conditions. In the dawn-dusk direction, we assume the Hall conductance to be uniform. This background ionospheric conductance is merely due to the solar contribution, and for simplicity, we assume it decreases linearly toward nightside. The solid line in the bottom panel of Figure 2 shows the background ionospheric potential distribution in the dawn-dusk cross section, which is identical for both runs. In the noonmidnight direction, the electrical potential is constant. For the purpose of comparison, the potential perturbation associated with the initial magnetospheric shear flow is shown by dashed line in the bottom panel. It can be seen that the distribution of background ionospheric potential in the bottom panel of Figure 2 implies a large-scale antisunward convection which is uniform in the dawn-dusk direction. The initial magnetospheric shear flow carried by the propagating Alfvén waves, which is identical for both runs, is represented by a Gaussian distribution potential as shown by the solid line in Figure 3. The shear flow is uniform in the noon-midnight direction. The corresponding electric field distribution, which is convergent in the center and divergent on the edges of the shear flow, is shown by the dashed line in Figure 3. This velocity shear is consistent with the overall feature of observed shear flows associated with polar cap arcs. It has been 
INITIAL BACKGROUND
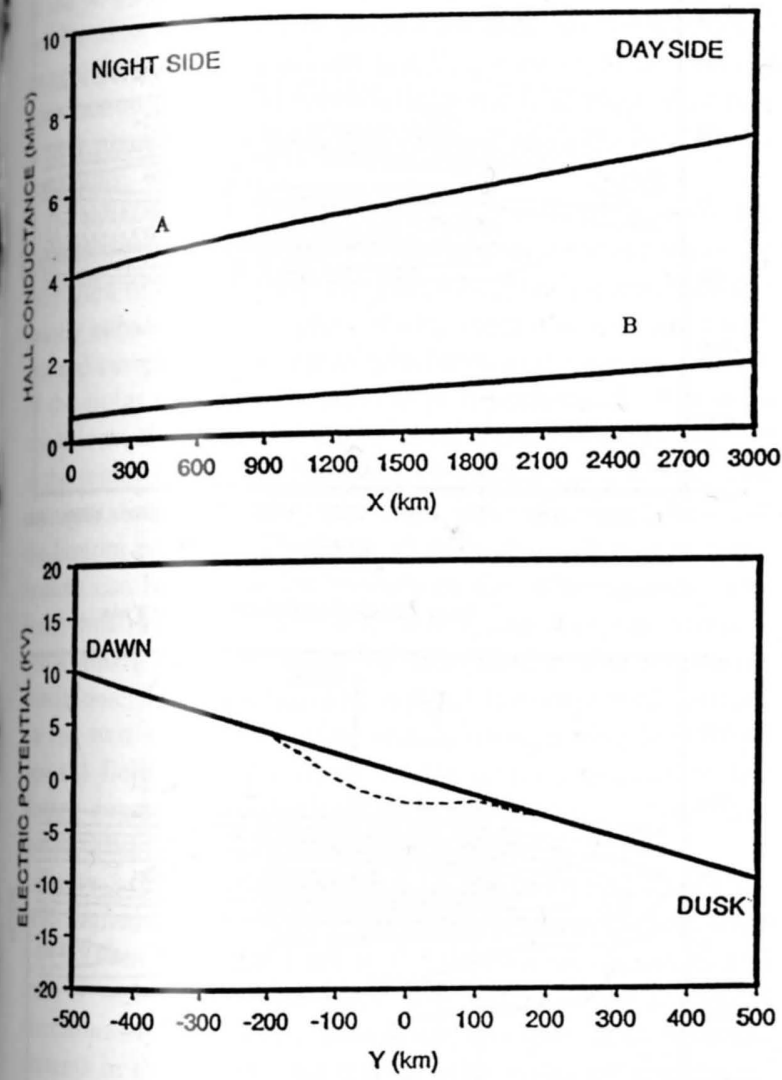

Fig. 2. Ionospheric background conditions. (a) The Hall conductance distribution in the noon-midnight cross section (top) in which curve $A$ denotes summer and curve B denotes winter values. (b) The ionospheric background potential distribution in the dawn-dusk cross section (bottom) in which the dashed line represents the potential perturbation associated with the initial magnetospheric shear flow.

observed that in many cases these shear flows and the associated arcs are very stable in time [e.g., Valladares and Carlson, 1991]. This might indicate that the shear flow adopted in this paper is stable, or at least the characteristic growth time for the fastest wave mode is still much longer than the characteristic time for the formation of polar cap arcs. For both runs, we assume that polar cap arcs occur on open field lines, which means the reflection coefficient at the magnetospheric boundary is - 1 everywhere in the calculation domain. A constant ratio between the Hall conductance and Pedersen conductance $\mathrm{R}=1.5$ is adopted as an approximate treatment. The time step is $10 \mathrm{~s}$, and the Alfvén wave traveling time between the magnetospheric boundary and ionosphere is assumed to be $2 \mathrm{~min}$. Other model parameters are as follows: thermal electron current $J_{O}=0.08 \mu \mathrm{A} \mathrm{m}^{-2}$; constant ionization coefficient $Q_{O}=7 \times 10^{-3}$ ions per electron Der meter; coefficient $\gamma^{\prime}$ defined in the prior section $=1 \times 10^{4}$
INITLAL PERTURBATION

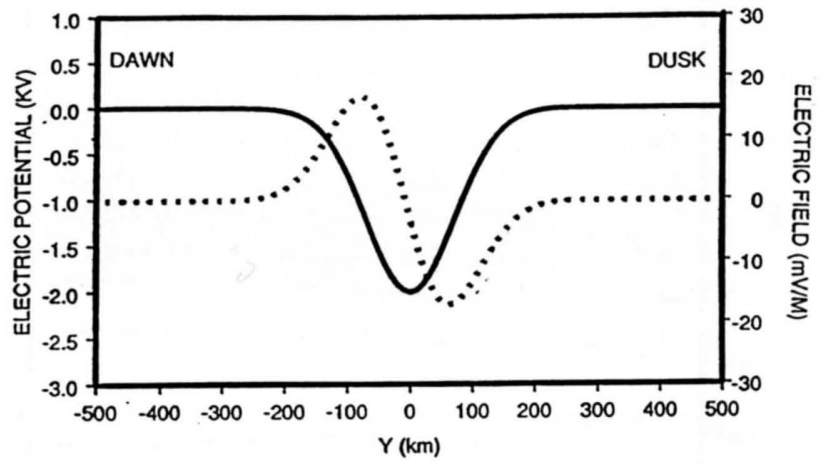

Fig. 3. Potential distribution in the dawn-dusk cross section associated with the initial magnetospheric shear flow (top) and the corresponding electric field distribution (bottom).

( ions m) / (electrons A) [Rees, 1963; Fridman and Lemaire, 1980]; and recombination coefficient $\beta=0.9 \times 10^{-13} \mathrm{~m}^{3} \mathrm{~s}^{-1}$ [Walls and Dunn, 1974].

The initial shear flow carried by Alfvén waves arrives in ionosphere at $\mathrm{t}=0 \mathrm{~min}$, which leads to the bouncing of Alfvén waves between the magnetosphere and ionosphere and the development of polar cap arcs. As long as the external environment of the coupled M-I system remains unchanged, the temporal change of the bouncing Alfvén waves will diminish with time due to the finite ionospheric conductance, and the development of polar cap arcs will approach an asymptotic steady state. Such a steady state of polar cap arcs is achieved in approximately 8 to $10 \mathrm{~min}$ in these runs. Figure 4 shows the asymptotic features of polar cap arcs at time $t=8 \mathrm{~min}$, in which the left column is for summer conditions while the right one is for winter conditions. The top panels of Figure 4 show the field-aligned current distribution in which dashed lines denote upward field-aligned currents, and solid lines denote downward field-aligned currents. Note that in order to display the detailed structures associated with polar cap arcs, only a portion of the simulation domain is shown in this figure. It can be seen that in the self-consistent development of a polar cap arc, a single peak precipitation associated with the initial magnetospheric shear flow has split into multiple peaks, leading to multiple structures of the polar cap arcs. This results might imply an important point that the observed multiple polar cap arcs are not due to any multiple structures in the magnetospheric source region, instead, such a multiple feature is internally originated in the dynamical process of polar cap arcs determined by the whole coupled M-I system. The major difference between the asymptotic fieldaligned current distributions associated with the arcs for summer and winter conditions is that the field-aligned currents for summer conditions have much stronger downward components on the dawn and dusk edges of the arcs. This is because of the lower background conductivity in winter preventing the upward field-aligned current in the arcs from flowing into the dark region outside of the arcs and connecting 

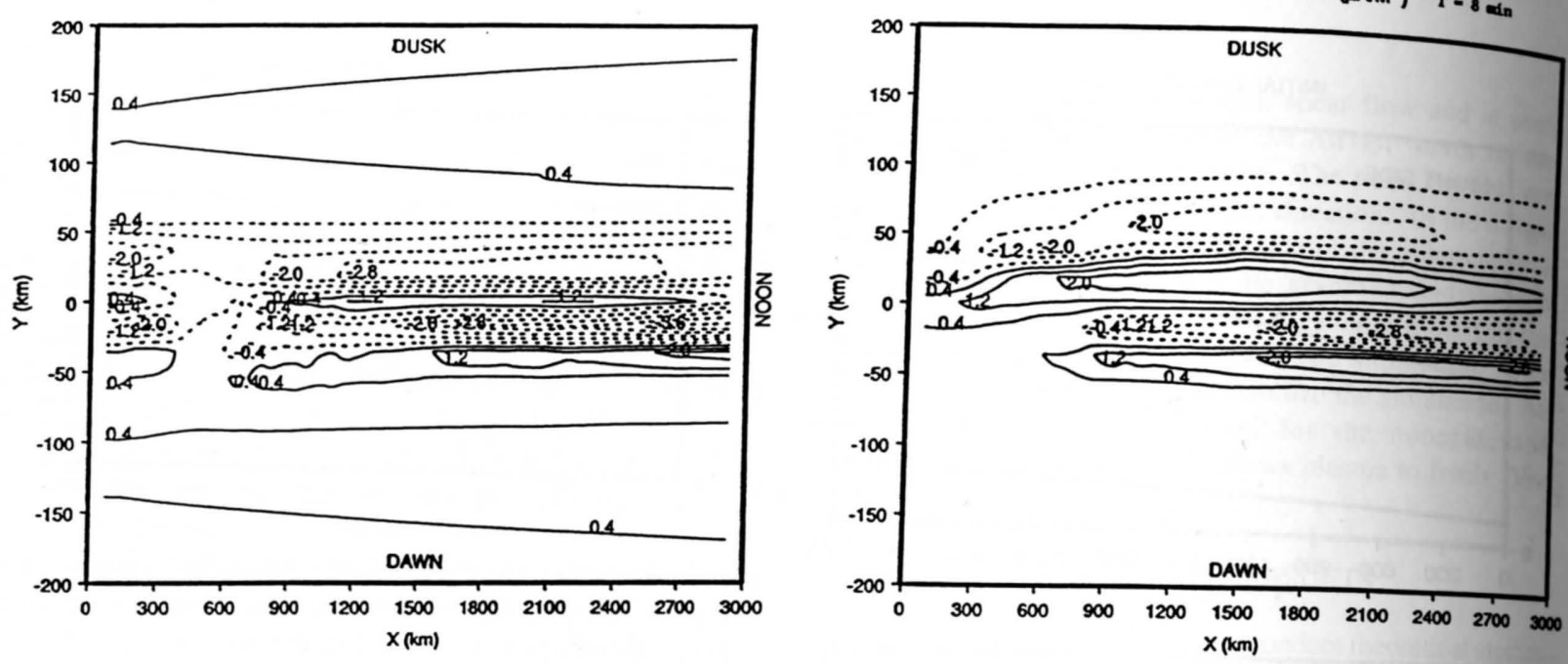

HALL CONOUCTANCE (MHO) T = 8 min

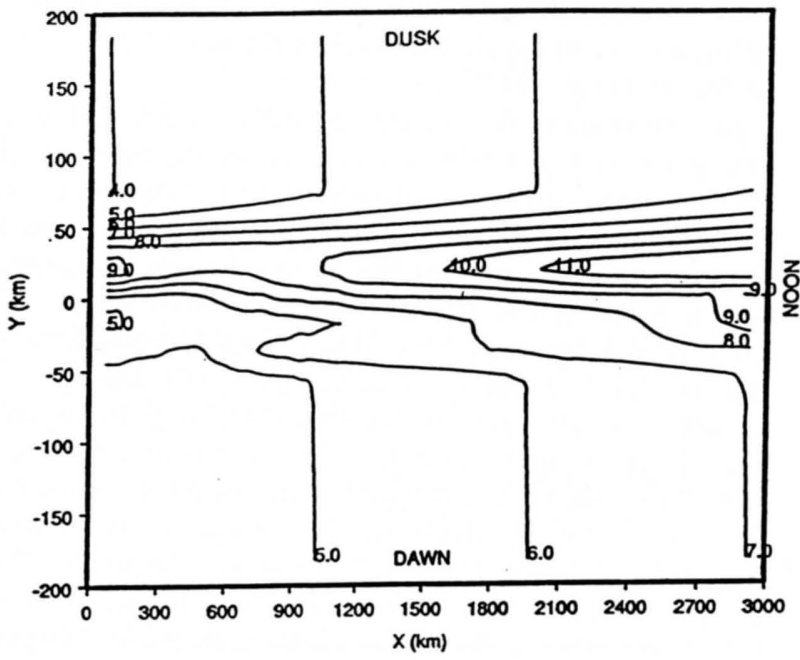

HALL CONDUCTANCE (MHO) $\mathrm{T}=8 \mathrm{~min}$

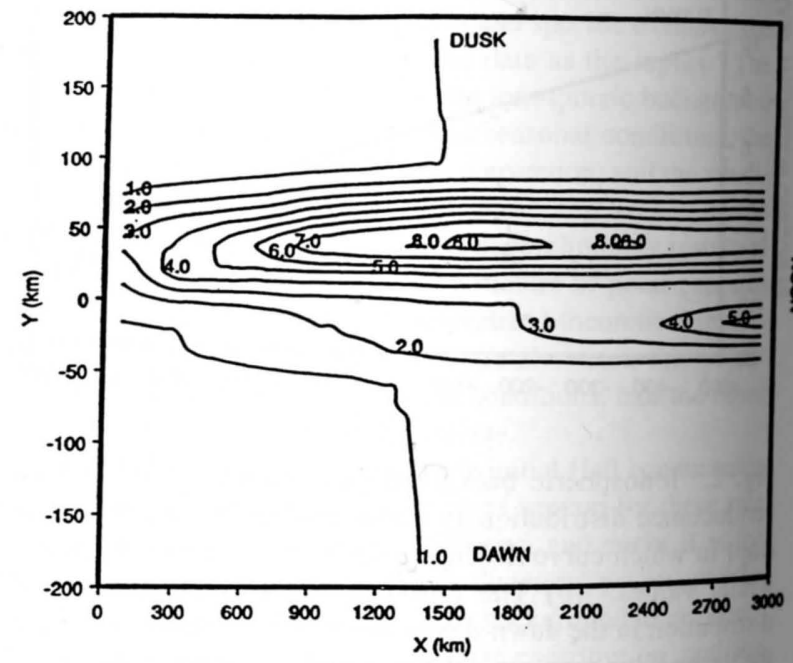

JOULE HEATING (ERG/CM ${ }^{2}$ SEC) T = 8 min

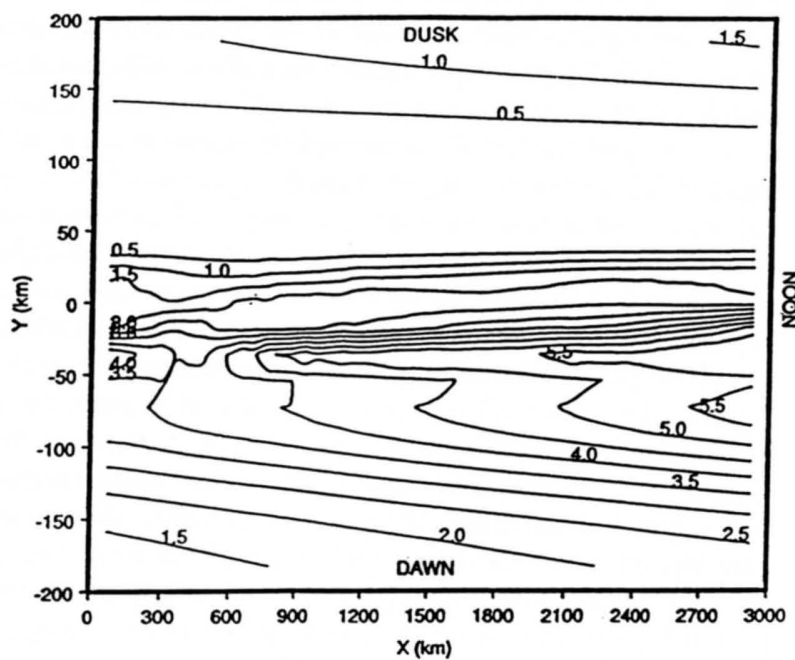

JOULE HEATING (ERG/CM ${ }^{2}$ SEC) T -8 min

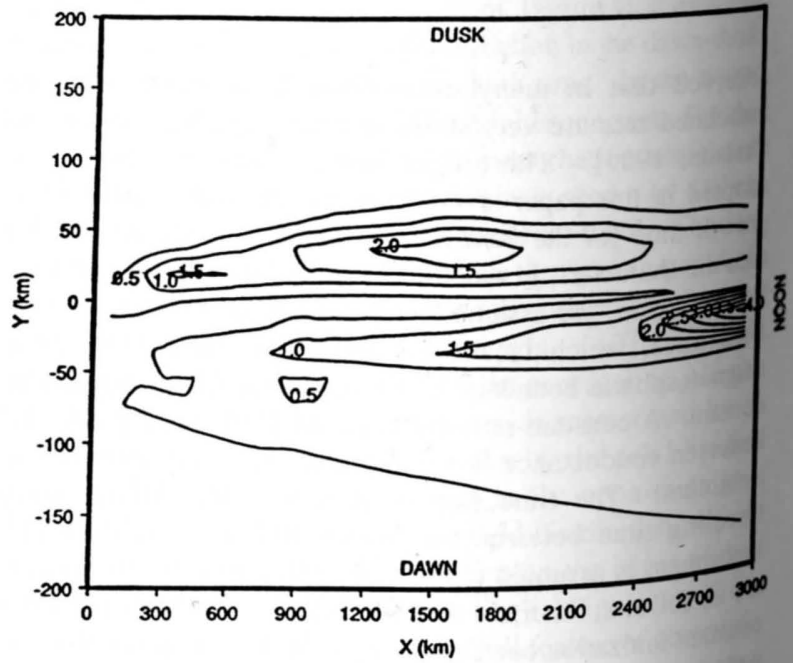


to the downward field-aligned currents there. Our simulation results also show that in polar cap arcs, the field-aligned current is mainly closed by the Pedersen current, and the contribution of the Hall component of the field-aligned current is only about $2 \%$. This is true for both summer and winter conditions.

The middle panels of Figure 4 show the distributions of the ionospheric Hall conductance at asymptotic steady state. In the region of the polar cap arcs, the ionospheric conductance is greatly enhanced by the precipitating electrons associated with upward currents, while the conductance in the regions outside of the polar cap arcs remains almost unchanged. For both conditions, the enhancement of conductivity is well confined in the regions bounded by strong electron precipitations. The asymptotic distributions of the Joule heating rate are shown in the bottom panels of Figure 4. A very sharp change in Joule heating can be seen in the vicinity of the polar cap arcs. The Joule heating associated with polar cap arcs for summer conditions is spatially extended compared to that for winter conditions. This is because in summer the horizontal currents on the two sides of arcs are much stronger due to a large upward field-aligned current in the arcs connecting to the distant downward field-aligned currents outside of the arcs, thereby leading to an extended heating region.

Figure 5 shows the evolution of total upward and downward field-aligned currents in the region which covers the range from $y=-200 \mathrm{~km}$ to $y=200 \mathrm{~km}$ in the dawn-dusk direction, and from $x=0 \mathrm{~km}$ to $x=3000 \mathrm{~km}$ in the noon-midnight direction. As expected, both total upward and downward currents in this region increase in time, while polar cap arcs are developing. One interesting feature shown in Figure 5 is the temporal variation of the difference between the total upward and total downward currents. In the early development stage of polar cap arcs, a substantial difference exists between the total upward and total downward currents. Since the Hall current is almost divergence-free, a significant amount of upward current in the arcs must connect to the distant ionosphere outside of the polar cap arcs via Pedersen current which is mainly in dawn-dusk direction. While polar cap arcs are developing, the conductance in the regions of the polar cap arcs is enhanced and less current flows into the lowerconducting regions outside of the polar cap arcs, thereby more upward current is closed by local downward current, and the total downward current catches up gradually. At the asymptotic steady state of the arcs, the difference between the total upward and total downward currents is almost zero. This indicates that while polar cap arcs are developing, the associated current system tends to become further localized, and at steady state the current system in the narrow regions around the arcs is locally self-closed. The greater increase of total field-aligned current for each bounce of the Alfvén wave in the summer case is due to a stronger wave reflection caused by a higher conductivity in summer.

Figure 6 shows the evolutions of the ratio between total Joule heating and total energy flux associated with precipitating electrons in the region around the polar cap arcs as described above. Initially, the ratio is much larger than 1 . When the polar cap arcs are fully developed, the ratio drops to less than one for both summer and winter conditions. A
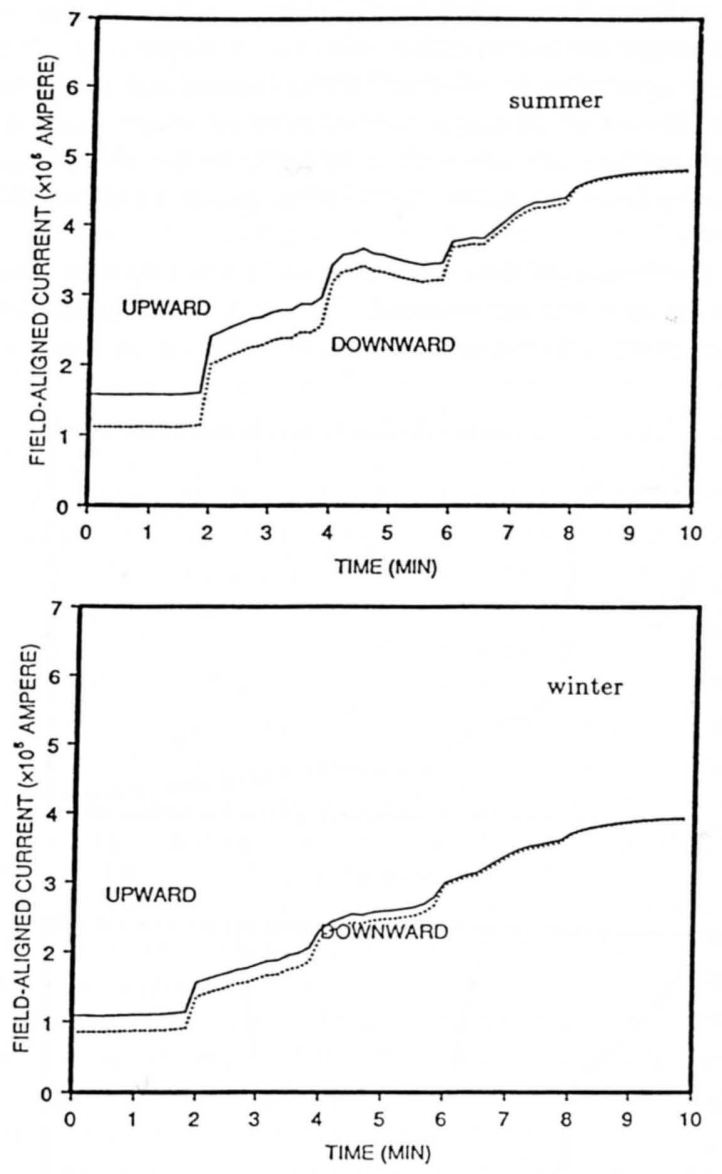

Fig. 5. Temporal evolution of the total upward and downward field-aligned currents in the region which covers the range from $y=-200 \mathrm{~km}$ to $y=200 \mathrm{~km}$ in the dawn-dusk direction, and from $x=0 \mathrm{~km}$ to $x=3000 \mathrm{~km}$ in the noon-midnight direction. The top panel shows values for summer conditions while the bottom panel shows values for winter conditions.

Fig. 4. The field-aligned current distributions (top) in which dashed lines denote the upward currents and solid lines denote the downward currents, Hall conductance distributions (middle), and Joule heating rates (bottom) at asymptotic steady state. The left column shows values for summer condition, while the right column shows values for winter conditions. 
commonly accepted fact is that in the polar cap, the Joule heating rate is much larger than the energy deposition from the precipitating electrons. However, our results suggest that this might not be true in the small-scale region around the polar cap arcs. A ratio of less than one can exist when the electric field is dramatically decreased, and precipitation is greatly enhanced in the narrow regions around the arcs.

The major results of these preliminary theoretical studies can be summarized as follows.

1. The time constant for the formation of polar cap arcs is around $10 \mathrm{~min}$.

2. In the self-consistent development of polar cap arcs, an initial single arc precipitation splits into multiple arcs. This result suggests that the observed multiple polar cap arcs might not be due to any multiple structures in the magnetospheric source region; instead, such a multiple feature is derived internally from the dynamical coupling processes of the M-I system.

3. Our model predicts the existence of plasma flow crossing polar cap arcs, but the amplitude of such a flow is small, and its time constant is much larger than that of polar cap arcs.
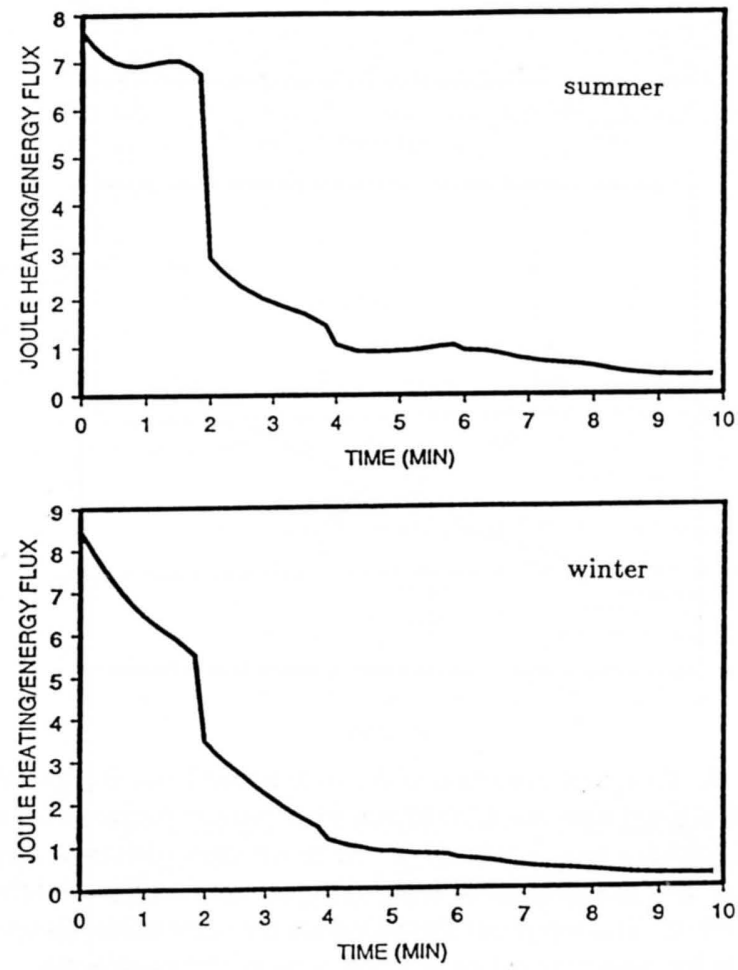

Fig. 6. Temporal evolution of the ratio between the total Joule heating and total energy flux associated with precipitating electrons in the region which covers the range from $y=-200 \mathrm{~km}$ to $y=200 \mathrm{~km}$ in the dawn-dusk direction, and from $x=0 \mathrm{~km}$ to $x=3000 \mathrm{~km}$ in the noon-midnight direction. The top panel shows values for summer conditions and the bottom panel shows values for winter conditions.
4. For summer conditions, the field-aligned currents associated with polar cap arcs have much stronger downward components on the dawn and dusk edges of the arcs, and the Joule heating region is much more extended.

5. While polar cap arcs are developing, the associated current system tends to become further localized and at steady state the current system in the narrow regions around the arcs is locally self-closed.

6. Because of the dramatically decreased electric field and greatly enhanced precipitation, the ratio between total Joule heating and total energy flux associated with precipitating electrons can be less than one in the narrow regions around polar cap arcs.

7. While polar cap arcs are developing, individual arcs are not spatially stable and they make dawn-dusk direction motions with an average velocity of around $200 \mathrm{~m} \mathrm{~s}^{-1}$.

\section{CAPABILITY AND POTENTIAL APPLICATIONS OF THE MODEL}

In this section, we describe the capability and potential applications of the time-dependent polar cap arc model in terms of two different research approaches, namely, the modelobservation study of specific features and specific events of polar cap arcs by directly using observational data as the inputs and the theoretical study of the typical or average features of the arcs by using the generalized theoretical inputs.

\subsection{Coordinated model-observation studies}

Several CEDAR-HLPS campaigns have lead to a very extensive observational database of polar cap arcs, including the data from all-sky cameras, sounding rockets, incoherent scatter radars, digisondes etc., and many new features of polar cap arcs have been observed during these campaigns. To quantitatively explain the observed new features and reveal the underlying physics and the associated electrodynamical processes, a series of model-observation studies with the focus on the specific new features of polar cap arcs would be a logical next step. The developed time-dependent model of polar cap arcs is suitable for such a coordinated effort.

The suitability of the model for the coordinated modelobservation study is reflected by the following features of the model. First, the model is time-dependent and electrodynamical. This means that with a set of observational data as the initial inputs, we can obtain a detailed picture of the temporal evolution of the electrodynamics associated with polar cap arcs. This time-dependent electrodynamical treatment also has a high temporal resolution of around $10 \mathrm{~s}$. In contrast, the conventional model-observation study of the arcs. in which a time sequence of data is needed to drive an ionosphere model and to obtain the temporal evolution of the arcs, has a poor temporal resolution restricted by the availability of the time sequence of data and is not 
electrodynamically self-consistent. Second, the time-dependent model of polar cap arcs treats the electrodynamics of polar cap arcs in the coupled magnetosphere-ionosphere system, and the interactions between the magnetosphere and the ionosphere and the active role played by the ionosphere are specifically stressed in this model.

To conduct model-observation study of specific features of the polar cap arcs, three major initial inputs need to be specified for the model. The first is the height-integrated background conductivity prior to the occurrence of the polar cap arcs; the second is the ionospheric background convection prior to the occurrence of polar cap arcs; and the last is the enhanced convection associated with polar cap arcs. These initial inputs can be directly and/or indirectly derived from CEDAR-HLPS observational database of polar cap arcs. With the initial inputs based on observations, the time-dependent model of polar cap arcs can provide the profiles of the temporal evolution of most of the electrodynamical parameters, that is, field-aligned current, electric field, ionospheric conductance, horizontal currents, Joule heating rate, etc. The spatial resolution for these profiles can be around $10 \mathrm{~km}$, and the temporal resolution can be about $10 \mathrm{~s}$.

The potential applications of the model for the modelobservation studies of several specific features of polar cap arcs observed during CEDAR-HLPS campaigns are listed in the following subsections.

4.1.1. Dawn-dusk motion of polar cap arcs. The dawn-dusk motion is an important dynamical feature of polar cap Sunaligned arcs observed during HLPS/GAPS campaigns. It is found by all-sky camera observations [Valladares, 1992] that the instantaneous speed of such a motion can be as high as $500 \mathrm{~m} \mathrm{~s}^{-1}$. Observations suggest that the dawn-dusk motion of polar cap arcs is probably IMF $B y$-related. Similar dawndusk motions of polar cap arcs have been observed in our simulations of polar cap arcs. Our preliminary results show that individual arcs are not spatially stable in time, and they move at an average speed of around $200 \mathrm{~m} \mathrm{~s}^{-1}$ in dawn-dusk direction, while their intensities vary.

A systematic model-observation study of the dawn-dusk motion of polar cap arcs can be done by using the developed model and observational data with the focus on finding out the controlling factors for the dawn-dusk motion of polar cap arcs and the physics behind them. A few typical events of polar cap arcs need to be selected first. The criterion for this selection will be mainly based on the dawn-dusk motion shown by all-sky camera data and the availability of the ISR $\left(E_{o}\right)$, With the background conductivity $\left(\Sigma_{o}\right)$, electric field $\left(E_{0}\right)$, and the enhanced electric field associated with polar cap arcs $(E)$, specified by the ISR data, the theoretical model can provide the time history of these polar cap arcs with a time resolution of $10 \mathrm{~s}$. The strength of this new approach is that the modeling can provide the temporal variations of the basic physical quantities which are relevant to the electrodynamics of polar cap arcs. Therefore the complete information of the be amical processes associated with the dawn-dusk motion can ained, and the comparison of the observed and modeled dawn-dusk motion can be conducted in an integrated dynamical picture.

Using the defined $\Sigma_{O}, E_{O}$, and $E$ and the results in the previous steps as a base, we then vary the initial conditions of the simulation in order to study the dawn-dusk motion in a more general fashion. The variation of the initial conditions will be based on the considerations of the effects of IMF, season, and the various magnetospheric drivers. These runs will provide the answers to a series of basic questions related to the dawn-dusk motion, such as (1) Is the motion IMF and/or seasonally dependent? (2) Is the motion due to the configuration change in the magnetosphere or internally determined by the ionosphere-magnetosphere dynamics? and (3) What are the controlling factors for the motion?

4.1.2. Rotation of ionospheric convection. One of the new features associated with polar cap arcs observed during HLPS/GAPS campaigns is that prior to the breakup of polar cap arcs, the adjacent convection appears to have a sudden rotation. This feature was observed by sounding rocket observations [Berg, 1992]. On the basis of our theoretical study, this feature might be due to the rotation of the reflected Alfvén wave field caused by the inhomogeneity of the ionosphere. Our results show that a strong conductivity nonuniformity in the direction, perpendicular to the incident wave field, large Hall and Pedersen conductivity ratios, and low conductivity values, lead to a large rotation of the reflected field. This general conclusion can be applied to the dynamics of polar cap arcs and a model-observation study of this rotational feature of the convection can be done to further validate the general theoretical conclusion and reveal the physics associated with this specific feature of polar cap arcs.

4.1.3. Multiple polar cap arc structures. The multiple structures of polar cap arcs have been confirmed by many observations, including those of all-sky cameras, during HLPS/GAPS campaigns. In addition to the ground-based observations, the existence of multiple structures of polar cap arcs has also been confirmed by satellite observations. It has been indicated by our theoretical simulations that the appearance of multiple structures in the polar cap arcs might not be due to any multiple structures in the magnetospheric source region; instead, such a multiple feature is internally generated in the dynamical processes of the coupled M-I system. A stronger ionospheric background convection leads to more structures in the arcs. This preliminary theoretical result shows the important role played by the ionosphere in the dynamics of polar cap arcs. As a follow-up of the observations and theoretical studies of the multiple polar cap arc structures, a model-observation study of this specific feature can be done to further reveal the underlying physics of the multiple structures in a more realistic approach.

\subsection{Theoretical modeling of typical and average features.}

In addition to the potential applications on the modelobservation study of specific features and/or specific events of 
polar cap arcs, the developed model can also be used to conduct simulations of typical and average features of polar cap arcs. It has been shown by observations that the feature of polar cap arcs has a strong dependence on the $Y$ component of IMF [e.g., Makita et al., 1991]. The seasonal and solar cycle variation of the ionospheric conditions might also influence the feature of polar cap arcs. The auroral oval regions are physically connected to polar cap arcs, and the precipitation in the auroral oval region could influence the polar cap arcs. A systematic theoretical modeling study using the developed time-dependent model of polar cap arcs could shed light on these important issues.

It has been shown that for various IMF conditions, the large-scale background ionospheric convection can be dramatically different [Heppner and Maynard, 1987]. By using various background convection patterns as model inputs, we can study the impact of global-scale convection, thereby the IMF, on the mesoscale features of polar cap arcs. The background convection patterns can be adopted from the empirical or statistical convection patterns (four-cell, threecell, or distorted two-cell). By varying the distribution of the background ionospheric conductivity, we can study the seasonal and solar cycle effects and the influence of auroral oval precipitation on the polar cap arcs. The variation of the background ionospheric conductivity can be obtained from theoretical static ionospheric models. We can also change the profile of the initial magnetospheric shear flow to study the effects of the characteristic energy of the precipitation and to test the effects of various magnetospheric drivers. The dependence upon magnetospheric topology can be modified by changing the bounce time and reflection coefficient. Indeed, a nonspatially homogeneous reflection coefficient can be used to better represent the different high-latitude magnetospheric regions.

Acknowledgment. This research was supported by NSF grant ATM-89-13230, NASA grant NAG5-1484, and grant AFOSR-90-0026 to Utah State University.

\section{REFERENCES}

Berg, G. A., In Situ and ground-based observations of Sunaligned arcs, paper presented at the Workshop for Coupling, Energetics, and Dynamics of Atmospheric Regions/HighLatitude Plasma Structures, June, 1992.

Carlson, H. C., Jr., V. B. Wickwar, E. J. Weber, J. Buchau, J. G. Moore, and W. Whiting, Plasma characteristics of polar cap F-layer arcs, Geophys. Res. Lett., 1, 895, 1984.

Carlson, H. C., R. A. Heelis, E. J. Weber, and J. R. Sharber, Mesoscale convection patterns during northward interplanetary magnetic field, J. Geophys. Res., 93, 14,501, 1988.

Crain, D. J., J. Sojka, R. Schunk, and L. Zhu, Modeling Sunaligned polar cap arcs, Radio Sci., 29, this issue.

Doe, R., M. Mendillo, J. Vickrey, M. Ruohoniemi, and R. Greenwald, Coordinated convection measurements in the vicinity of auroral cavities and arcs, Radio Sci., 29, this
issue.

Frank, L. A., J. D. Craven, D. A. Gurnett, S. D. Shawhan, D. R. Weimer, J. L. Burch, J. D. Winningham, C. R Chappell, J. H. Waite, R. A. Heelis, N. C. Maynard, M Sugiura, W. K. Peterson, and E. G. Shelley, The theta aurora, J. Geophys. Res., 91, 3177, 1986.

Fridman, M., and J. Lemaire, Relationship between auroral electron fluxes and field-aligned electric potential difference J. Geophys. Res., 85, 664, 1980.

Hardy, D. A., W. J. Burke, and M. S. Gussenhoven, DMSP optical and electron measurements in the vicinity of polar cap arcs, J. Geophys. Res., 87, 2413, 1982.

Heppner, J. P., and N. C. Maynard, Empirical high-latitude electric field models, J. Geophys. Res., 92, 4467, 1987.

Makita, K, C.-I. Meng, and S.-I. Akasofu, Transpolar auroras, their particle precipitation, and IMF component, J. Geophys. Res., 96, 14,085, 1991.

Nielsen, E., J. D. Craven, L. A. Frank, and R. A. Heelis, Ionospheric flows associated with a transpolar arc, J. Geophys. Res., 95, 21169, 1990.

Obara, T., M. Kitayama, T. Mukai, N. Kaya, J. S. Murphree, and L. L. Cogger, Simultaneous observations of Sun-aligned polar cap arcs in both hemispheres by EXOS-C and Viking, Geophys. Res. Lett., 15, 713, 1988.

Rees, M. H., Auroral ionization and excitation by incident energetic electrons, Planet. Space. Sci., 11, 1209, 1963.

Rich, F. J., D. A. Hardy, R. H. Redus, and M. S. Gussenhoven, Northward IMF and patterns of high-latitude precipitation and field-aligned currents: The February 1986 storm, J. Geophys. Res., 95, 7893, 1990.

Schunk, R. W., and J. J. Sojka, Ion temperature variations in the daytime high-latitude F region, J. Geophys. Res., 87, 5169, 1982.

Valladares, C. E., Observations of polar cap Sun-aligned arc electrodynamics, paper presented at the Workshop for Coupling Energetics and Dynamics of Atmospheric Regions High-Latitude Plasma Structure, June, 1992.

Valladares, C. E., and H. C. Carlson, The electrodynamic, thermal, and energetic character of intense Sun-aligned arcs in the polar cap, J. Geophys. Res., 96, 1379, 1991.

Walls, F. L., and G. H. Dunn, Measurement of total crosssection for electron recombination with $\mathrm{NO}^{+}$and $\mathrm{O}_{2}^{-}$using ion storage technology, J. Geophys. Res., 79, 1911, 1974.

Weber, E. J., and J. Buchau, Polar cap F-layer auroras, Geophys. Res. Lett., 8, 125, 1981.

Zhu., L., J. J. Sojka, R. W. Schunk, and D. J. Crain, A timedependent model of polar cap arcs, J. Geophys. Res., 98, 6139-6150, 1993.

D. J. Crain, R. W. Schunk, J. J. Sojka, and L. Zhu. Center for Atmospheric and Space Sciences, Utah State University, Logan, UT 84322-4405.

(Received November 30, 1992; revised March 8, 1993, accepted April 20, 1993) 